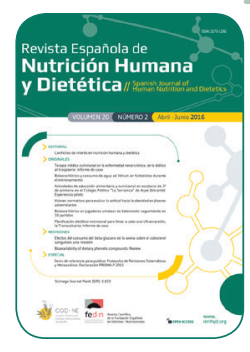

\title{
ORIGINAL
}

\section{Balance hídrico en jugadoras amateur de baloncesto: seguimiento en 10 partidos}

\author{
Abraham Batalla-Gavalda ${ }^{a}$, Ana Maria Bofill-Ródenas ${ }^{b}$, Francisco Corbi-Soler ${ }^{c}$, Raúl Montoliu-Colás , \\ Antoni Planas-Anzano ${ }^{C, *}$
}

a Departamento de Salud y Gestión, INEFC de Lleida, Universidad de Lleida, España.

b Departamento de Ciencias Médicas, Facultad de Medicina, Universidad de Girona, España.

C Grupo de Investigación del Movimiento Humano, INEFC de Lleida, Universidad de Lleida, España.

d Instituto de Nuevas Tecnologías de la Imagen (INIT), Universidad Jaume I, Castelló, España.

*f@corbi.neoma.org

Recibido el 23 de diciembre de 2015; aceptado el 23 de febrero de 2016.

Balance hídrico en jugadoras amateur de baloncesto: seguimiento en 10 partidos

\section{PALABRAS CLAVE \\ Equilibrio hidroelectrolítico; \\ Deshidratación; \\ Baloncesto; \\ Mujeres.}

\section{RESUMEN}

Introducción: El objetivo del presente estudio fue analizar la evolución de las necesidades hídricas de un equipo de baloncesto femenino amateur, en situación de partido y durante toda una fase eliminatoria, a partir de la pérdida de peso experimentada. Además, se analizó la existencia de posibles diferencias en el nivel de deshidratación en función de la posición de juego.

Material y Métodos: Estudio piloto de un solo grupo con mediciones antes de la competición y después de la competición. Jugadoras amateurs de baloncesto $(n=10)$ y sus recipientes de rehidratación fueron pesadas antes y después de los 10 partidos analizados. Las variaciones de peso fueron evaluadas teniendo en cuenta el líquido ingerido de los bidones de reposición y el líquido evacuado a través de la orina.

Resultados: Los resultados de este estudio sugieren la existencia de diferencias significativas entre el peso corporal antes y después de cada partido $(z=8,551 ; p<, 0005)$. La magnitud de estas diferencias parece ser muy distinta en función del partido y la jugadora analizada, con valores medios que oscilan entre los $0,63 \mathrm{~kg}(0,9 \%$ Peso Corporal) y los 0,95kg (1,37\% Peso Corporal) de pérdida de peso corporal. Además, no se observaron diferencias significativas en el nivel de deshidratación en función de la posición de juego ( $F=1,59 ; p=0,1929)$.

Conclusiones: Se confirma la existencia de una alta variabilidad intra e interpersonal en cuanto a la pérdida de masa corporal durante los 10 partidos analizados, lo que sugiere la necesidad de una monitorización y rehidratación individualizada. 
Fluid balance in amateur female basketball players: follow-up of 10 games

\section{KEYWORDS}

Water-Electrolyte Balance;

Dehydration;

Basketball;

Women.

\begin{abstract}
Introduction: The aim of the present study was to understand the development of hydration needs during a playoff stint, in a match situation, for an amateur women's basketball team. In addition, the existence of possible differences in the level of dehydration according to playing position was analyzed.
\end{abstract}

Material and Methods: Pilot study with single group measurements before competition and after competition was done. Both amateur basketball players $(n=10)$ and their personal water containers were weighted before and after every match. Weight variations were evaluated considering the ingested liquid and replenishment liquid discharged through urine.

Results: Results showed that there are significant differences between body mass before and after each match $(z=8.551 ; p<.0005)$. However, magnitude of dehydration was very different in each player and match, with average values between $0.63 \mathrm{~kg}(0.9 \%$ body weight) and $0.95 \mathrm{~kg}$ (1.37\% body weight). In addition, there were not significant differences in the level of dehydration according to the playing position ( $F=1.59 ; p=0.1929)$.

Conclusions: These evaluations confirm high intra and interpersonal variations as per body mass loss during the 10 referred playoff stint matches. Recommendation of creation of individualized hydration protocols is suggested.

\section{CITA}

Batalla-Gavalda A, Bofill-Ródenas AM, Corbi-Soler F, Montoliu-Colás R, Planas-Anzano A. Balance hídrico en jugadoras amateur de baloncesto: seguimiento en 10 partidos. Rev Esp Nutr Hum Diet. 2016; 20(2): 111 - 119. doi: 10.14306/renhyd.20.2.204

\section{INTRODUCCIÓN}

Una correcta hidratación resulta fundamental a la hora de optimizar el rendimiento deportivo, prevenir la aparición de lesiones y recuperar el equilibrio corporal. Diversos son los mecanismos a través de los cuales el cuerpo tiende a la deshidratación. Los más importantes son la sudoración, la transpiración insensible, la orina, las heces, la digestión y la respiración. Además, la deshidratación puede verse incrementada por numerosos factores como son las condiciones ambientales, la dieta, la composición corporal, la ingesta de determinados medicamentos o suplementos deportivos, el nivel de aclimatación y tolerancia al calor, el momento de la temporada, la cantidad y tipo de actividad física, los hábitos sociales y los niveles de hidratación pre$\mathrm{via}^{1}$. Diversos estudios sugieren que los niveles normales de hidratación fuera de la práctica deportiva son en general incorrectos, constatándose en algunos casos que el deportista empieza la práctica deportiva en deuda hídrica²
Todo ello podría tener repercusiones negativas, tanto en el rendimiento deportivo como en el estado de salud del deportista, ya que pequeños niveles de deshidratación, entre el $1 \%$ y el $2 \%$ del peso corporal (PC) parecen comprometer seriamente la función fisiológica y el rendimiento durante la práctica deportiva ${ }^{3,4}$. Por todo ello, la valoración del estado hídrico del deportista resulta fundamental.

Diversas son las metodologías que habitualmente se utilizan para la valoración de los procesos de deshidratación: la valoración de los niveles de hematocrito, hemoglobina, plasma, sodio, potasio y magnesio en sangre ${ }^{5}$; el control de la cantidad, color y concentración de sustratos en la orina ${ }^{6}$; la fluctuación de determinadas hormonas en sangre como la aldosterona7 o la presencia y concentración de sustratos en la piel. Desafortunadamente, este tipo de metodologías implican un coste económico demasiado elevado y requieren en ocasiones de un personal sanitario especializado que se encargue de la obtención y tratamiento de las muestras. 
Por otro lado, la monitorización del peso corporal está aceptada como una forma válida, simple y no invasiva de detectar las diferencias hídricas entre antes y después del ejercicio. En este sentido, la National Athletic Trainers' Association $^{3}$ recomienda calcular la tasa de sudoración para conocer el nivel de deshidratación del sujeto. La tasa de sudoración se define como la relación entre el peso corporal, la ingesta de líquidos y el volumen de orina excretado durante la práctica del ejercicio ${ }^{7}$.

Conscientes de la importancia de conocer los niveles de deshidratación durante la práctica deportiva, el objetivo del presente estudio fue analizar la modificación del peso corporal, como reflejo del nivel de hidratación, en un grupo de jugadoras de baloncesto femenino amateur durante diez partidos de competición pertenecientes a una fase eliminatoria.

\section{MATERIAL Y MÉTODOS}

La muestra de este estudio estuvo formada por jugadoras $(n=10)$ pertenecientes a un mismo equipo de baloncesto competitivo amateur de nivel nacional. Los criterios de inclusión fueron: participar en un mínimo de un $80 \%$ de los entrenamientos y competiciones de su equipo; no recibir recompensa económica o en especie por su colaboración; no estar tomando ningún tipo de medicación; no seguir ningún tipo de dieta alimenticia, ni ninguna otra actividad que pueda alterar su función metabólica o respiratoria. Este estudio se diseñó teniendo en cuenta los principios de la Declaración de Helsinki. El estudio fue aprobado por el comité de ética local.

Procedimiento e instrumentos: En primer lugar, se registraron los valores antropométricos (peso, talla y porcentaje de grasa), realizando para ello una sola medición de cada una de las jugadoras participantes en este estudio. Para la determinación del peso, se utilizó una báscula de columna Seca ${ }^{\circledR} 700$ (Alemania) con una precisión de $0,05 \mathrm{~kg}$ y un tallímetro Seca ${ }^{\circledR} 220$, con una precisión de 0,1cm integrado en la báscula. Para la valoración del porcentaje de grasa, se utilizó el medidor: HBF-306-E (Omron Healthcare Europe, B.V) con un error de $0,1 \%$.

Para el cálculo del balance hídrico, las jugadoras fueron pesadas en una báscula Siltec Large Capacity Model GS-1 (Ohaus, Ohio, USA), antes y después de cada uno de los 10 partidos de competición oficial. A cada una de ellas, se le asignó un recipiente individual de rehidratación que fue también pesado, antes y después de cada partido con una báscula de precisión Traveler TA5000 (Ohaus, Ohio, USA). Todos los instrumentos fueron calibrados antes de cada partido siguiendo las indicaciones del fabricante. No se ingirieron alimentos en las dos horas previas al inicio de cada partido. Durante su transcurso las jugadoras sólo ingirieron agua a temperatura ambiente, ad libitum y sin la existencia de ningún protocolo de hidratación. Aunque algunos autores han advertido del riesgo de sufrir hiponatremia al ingerir bebidas bajas en sodio ${ }^{8}$, éste era el tipo de líquido que normalmente ingerían las participantes en el estudio durante la práctica deportiva habitual. Si por alguna razón, los bidones de reposición fueron rellenados durante la competición por alguno de los entrenadores, uno de los miembros del equipo de investigación se ocupó de medir y registrar las cantidades administradas. Además, durante los tiempos muertos y las pausas entre cuartos no se permitió a las jugadoras que utilizarán ningún método artificial de refrigeración con la intención de mejorar su termorregulación, tales como duchas de agua fría, mojarse la cabeza, nuca o muñecas o utilizar chalecos de enfriamiento. En el caso de requerir orinar, las jugadoras fueron pesadas antes y después de acudir al servicio.

Los cambios de peso a lo largo del partido y la cantidad de líquido consumido se utilizaron para calcular el nivel de hidratación a partir de la fórmula propuesta por Cox:

\section{Pérdida de líquido $(\mathrm{kg})=$}

Masa de la jugadora antes el partido (kg) - Masa de la jugadora después del partido $(\mathrm{kg})+$ Líquido ingerido $(\mathrm{kg})$ - Orina o Heces expulsadas $(\mathrm{kg})$

Posteriormente, los resultados fueron expresados en tantos por ciento, en relación al peso de cada jugadora. No se tuvieron en consideración en este estudio las posibles ganancias de masa corporal a partir de los fluidos absorbidos a través de la piel en situaciones de humedad.

La media de los datos ambientales de temperatura, humedad relativa y de la velocidad del viento durante los 10 partidos fueron tomados con una estación meteorológica portátil Krestel ${ }^{\circledR}$ K4500 (Boothwin, PA.)

Análisis de los datos: En el análisis estadístico, en primer lugar se realizó un análisis descriptivo de los datos. Seguidamente, se comprobó la normalidad de las variables "Peso inicial", "Peso final" y "posición de juego" mediante la prueba de Shapiro-Wilk (SW). En el caso de que no se cumplieran los supuestos de normalidad, se comparó el "Peso inicial" y el "Peso final" mediante la prueba de Wilcoxon. Las diferencias fueron consideradas estadísticamente significativas cuando $p<0,05$. Para la realización de todos los cálculos se utilizó el programa estadístico SPSS $\Theta_{\text {, versión } 15 .}$ 


\section{RESULTADOS}

Las características individuales de los sujetos participantes en este estudio pueden ser consultadas en la Tabla 1, mientras que las condiciones ambientales en las que se realizó el estudio se presentan en la Tabla 2.
En relación a las pérdidas de peso, en primer lugar se analizó la normalidad de los datos, obteniéndose vulneraciones estadísticamente significativas ("Peso inicial"; $S W=0,212$; $p<, 0005 ;$ "Peso final"; $S W=0,214 ; p<, 0005)$, lo que confirma que la muestra no cumplía los criterios de normalidad. Seguidamente, se valoró la existencia de diferencias significativas entre el peso corporal valorado antes y después de

Tabla 1. Características antropométricas (media y desviación estándar) de los sujetos participantes en el estudio.

\begin{tabular}{l|c|c|c|c|c} 
Participante & $\begin{array}{c}\text { Edad } \\
\text { (años) }\end{array}$ & $\begin{array}{c}\text { Talla } \\
(\mathrm{m})\end{array}$ & $\begin{array}{c}\text { Peso } \\
(\mathrm{kg})\end{array}$ & $\begin{array}{c}\text { Grasa Corporal } \\
(\%)\end{array}$ \\
\hline 1 & 19 & 1,79 & 65,5 & 16,8 \\
2 & 24 & 1,68 & 50,7 & 14,9 \\
3 & 20 & 1,85 & 95,6 & 26,7 \\
4 & 22 & 1,80 & 66,6 & 21,3 \\
5 & 21 & 1,67 & 67,2 & 23,7 \\
6 & 18 & 1,84 & 65 & 19,5 \\
7 & 24 & 1,88 & 73,8 & 18,5 \\
8 & 18 & 1,82 & 67,8 & 22,7 \\
9 & 26 & 1,70 & 64,5 & 20,1 \\
10 & 21 & 1,75 & 71,7 & 23,2 \\
Media & 21,3 & $\mathbf{1 , 7 7 8}$ & $\mathbf{6 8 , 8 4}$ & $\mathbf{2 0 , 7 4}$ \\
DE & $\mathbf{2 , 7 1}$ & $\mathbf{0 , 0 7 4}$ & $\mathbf{1 1 , 2 1}$ & $\mathbf{3 , 5 1}$ \\
& & & &
\end{tabular}

DE: Desviación Estándar.

Tabla 2. Media y desviación estándar de la temperatura, humedad relativa y velocidad del viento de cada uno de los 10 partidos analizados en este estudio.

\begin{tabular}{l|c|c|c|c|c|c|c|c|c|c} 
Partido (P) & P1 & P2 & P3 & P4 & P5 & P6 & P7 & P8 & P9 & P10 \\
\hline Temperatura (oC) & 9,16 & 15,07 & 8,95 & 12,07 & 11,47 & 13,57 & 15,97 & 24,83 & 12,65 & 14,03 \\
& $(0,59)$ & $(1,83)$ & $(0,81)$ & $(0,38)$ & $(0,38)$ & $(0,66)$ & $(0,42)$ & $(0,31)$ & $(0,67)$ & $(0,28)$ \\
\hline Humedad relativa (\%) & 82,65 & 45,50 & 72,83 & 77,67 & 77,5 & 63,33 & 54,83 & 31,17 & 69,67 & 83,17 \\
& $(2,20)$ & $(11,59)$ & $(1,32)$ & $(2,50)$ & $(1,38)$ & $(1,51)$ & $(2,79$ & $(0,75)$ & $(3,78)$ & $(2,48)$ \\
\hline Velocidad viento (km/h) & 1,11 & 3,25 & 1,85 & 1,33 & 1,20 & 1,70 & 2,30 & 2,62 & 1,58 & 0,95 \\
& $(0,31)$ & $(0,82)$ & $(0,51)$ & $(0,58)$ & $(0,47)$ & $(0,83)$ & $(0,22)$ & $(0,26)$ & $(0,21)$ & $(0,61)$
\end{tabular}


cada partido ( $z=8,551 ; p<, 0005)$, lo que sugiere la existencia de un proceso de deshidratación a lo largo de los 10 partidos analizados.

Al analizar los valores individuales y por partido, se observó una alta variabilidad en los resultados obtenidos, observándose valores que alcanzan el 1,94\% de pérdida del PC. Los valores medios obtenidos, tanto en $\mathrm{kg}$ como en porcentaje de pérdida de $\mathrm{PC}$, se situaron entre el $0,83 \%$ y el $1,57 \%$ del PC (Tabla 3).

Finalmente, se analizaron los datos en función de la posición de juego, no observándose diferencias significativas ( $F=1,59 ; \mathrm{p}=0,1929$ ) (Tabla 4).

Además, en relación a las pérdidas individuales se constataron grandes diferencias entre jugadoras, así como la existencia de una gran variabilidad intrapersonal al comparar los diferentes partidos analizados (Figura 1).

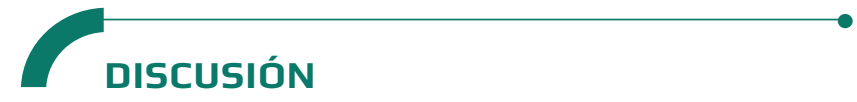

En general, se considera que la deshidratación provoca efectos negativos en los sistemas cardiovascular, termorregulador, metabólico y endocrino, pudiendo acabar generando la aparición de fatiga y una disminución del rendimiento ${ }^{10}$.

En los deportes colectivos, las necesidades hídricas vienen determinadas por diversos factores como el tipo de modalidad deportiva, el rol adoptado durante el juego, el período de la temporada, y el tipo de entrenamiento ${ }^{11}$. En general, se acepta que la deshidratación posee mayores efectos en aquellas actividades de alta intensidad en la que también tiene un papel fundamental la resistencia. Así por ejemplo, podemos citar deportes como el tenis, el fútbol, el voleibol o el baloncesto ${ }^{12-13}$. Además, muchos de estos deportes se desarrollan en condiciones ambientales caracterizadas por altos niveles de temperatura y humedad relativa que facilitan el proceso de deshidratación.

Tabla 3. Datos individuales de la modificación del porcentaje de PC de cada una de las jugadoras por partido. En la parte inferior media \pm desviación estándar, tanto en kg como en porcentaje de PC.

\begin{tabular}{|c|c|c|c|c|c|c|c|c|c|c|c|}
\hline Jugadora / Partido & P1 & P2 & P3 & P4 & P5 & P6 & P7 & P8 & P9 & P10 & Media \\
\hline $\mathrm{J1}$ & $-0,61$ & $-0,23$ & $-0,16$ & $-0,32$ & $-0,28$ & $-0,89$ & $-0,95$ & $-0,57$ & $-0,78$ & $-0,87$ & $-0,56$ \\
\hline 32 & $-0,79$ & $-0,64$ & $-0,85$ & $-0,58$ & $-0,48$ & $-0,94$ & $-0,62$ & $-0,67$ & $-1,43$ & $-0,98$ & $-0,80$ \\
\hline J3 & $-0,99$ & $-1,01$ & $-0,88$ & $-0,75$ & $-0,73$ & $-0,85$ & NO DATA & $-0,77$ & $-0,43$ & $-0,98$ & $-0,82$ \\
\hline 34 & $-0,86$ & $-0,52$ & $-0,88$ & $-1,07$ & $-0,71$ & $-0,83$ & $-0,57$ & $-0,40$ & $-0,50$ & $-0,50$ & $-0,68$ \\
\hline 35 & $-0,98$ & $-0,75$ & $-0,85$ & $-0,73$ & $-0,57$ & $-0,66$ & NO DATA & NO DATA & $-1,21$ & $-0,88$ & $-0,83$ \\
\hline J6 & $-0,58$ & $-1,08$ & $-0,69$ & $-0,52$ & $-0,81$ & $-0,79$ & $-0,58$ & $-0,49$ & $-0,97$ & $-0,85$ & $-0,74$ \\
\hline 37 & $-1,94$ & $-0,65$ & $-1,59$ & $-1,38$ & $-0,83$ & $-1,02$ & $-0,83$ & $-1,34$ & $-0,99$ & $-1,40$ & $-1,20$ \\
\hline 38 & $-0,53$ & $-0,78$ & $-0,92$ & $-0,74$ & $-0,62$ & $-1,01$ & $-0,85$ & $-0,97$ & $-0,72$ & $-0,90$ & $-0,81$ \\
\hline J9 & $-0,86$ & $-0,53$ & $-0,71$ & $-1,10$ & $-0,35$ & $-0,67$ & $-0,96$ & $-0,74$ & $-1,33$ & $-1,26$ & $-0,85$ \\
\hline $\mathrm{j} 10$ & $-0,68$ & $-0,58$ & $-1,27$ & $-1,25$ & $-0,91$ & $-0,63$ & $-1,08$ & $-1,17$ & $-0,82$ & $-0,84$ & $-0,92$ \\
\hline Media & $-0,88$ & $-0,68$ & $-0,88$ & $-0,84$ & $-0,63$ & $-0,83$ & $-0,80$ & $-0,79$ & $-0,92$ & $-0,95$ & $-0,82$ \\
\hline kg (\%PC) & 0,83 & 1,54 & 0,85 & 0,99 & 1,19 & 1,12 & 1,57 & 1,17 & 1,30 & 1,27 & 1,18 \\
\hline Media+DE & $-1,29$ & $-0,92$ & $-1,25$ & $-1,19$ & $-0,84$ & $-0,97$ & $-1,00$ & $-1,10$ & $-1,25$ & $-1,19$ & $-0,99$ \\
\hline kg (\%PC) & 1,27 & 2,08 & 1,03 & 1,30 & 1,48 & 1,42 & 2,09 & 1,39 & 1,78 & 1,61 & 1,54 \\
\hline Media-DE & $-0,48$ & $-0,43$ & $-0,51$ & $-0,50$ & $-0,42$ & $-0,69$ & $-0,61$ & $-0,48$ & $-0,58$ & $-0,70$ & $-0,65$ \\
\hline kg (\%PC) & 0,39 & 1,00 & 0,66 & 0,68 & 0,90 & 0,82 & 1,05 & 0,94 & 0,82 & 0,92 & 0,82 \\
\hline
\end{tabular}


Rev Esp Nutr Hum Diet. 2016; 20(2): 111 - 119

Balance hídrico en jugadoras amateur de baloncesto: seguimiento en 10 partidos

Tabla 4. Modificación del \% de PC en función de la posición de juego, con una muestra de dos jugadoras por posición.

\begin{tabular}{|c|c|c|c|c|c|c|c|c|c|c|c|}
\hline Posición\Partido & P1 & P2 & P3 & P4 & P5 & P6 & P7 & P8 & P9 & P10 & Media \\
\hline Base & $\begin{array}{l}-0,89 \\
(0,13)\end{array}$ & $\begin{array}{l}-0,69 \\
(0,08)\end{array}$ & $\begin{array}{l}-0,85 \\
(0,00)\end{array}$ & $\begin{array}{l}-0,65 \\
(0,11)\end{array}$ & $\begin{array}{c}-0,52 \\
(0,06)\end{array}$ & $\begin{array}{l}-0,80 \\
(0.20)\end{array}$ & $\begin{array}{c}-0,31 \\
(---)\end{array}$ & $\begin{array}{c}-0,34 \\
(---)\end{array}$ & $\begin{array}{l}-1,32 \\
(0,16)\end{array}$ & $\begin{array}{c}-0,93 \\
(0,07)\end{array}$ & $\begin{array}{c}-0,73 \\
(0,10)\end{array}$ \\
\hline Escolta & $\begin{array}{l}-0,73 \\
(0,18)\end{array}$ & $\begin{array}{l}-0,38 \\
(0,21)\end{array}$ & $\begin{array}{l}-0,43 \\
(0,39)\end{array}$ & $\begin{array}{c}-0,71 \\
(0,55)\end{array}$ & $\begin{array}{l}-0,32 \\
(0,05)\end{array}$ & $\begin{array}{l}-0,78 \\
(0,16)\end{array}$ & $\begin{array}{l}-0,95 \\
(0,01)\end{array}$ & $\begin{array}{l}-0,65 \\
(0,12)\end{array}$ & $\begin{array}{l}-1,06 \\
(0,39)\end{array}$ & $\begin{array}{l}-1,07 \\
(0,28)\end{array}$ & $\begin{array}{l}-0,71 \\
(0,23)\end{array}$ \\
\hline Alero & $\begin{array}{l}-0,63 \\
(0,07)\end{array}$ & $\begin{array}{l}-0,83 \\
(0,35)\end{array}$ & $\begin{array}{l}-0,98 \\
(0,41)\end{array}$ & $\begin{array}{l}-0,89 \\
(0,52)\end{array}$ & $\begin{array}{l}-0,86 \\
(0,07)\end{array}$ & $\begin{array}{c}-0,71 \\
(0,11)\end{array}$ & $\begin{array}{c}-0,83 \\
(0,35)\end{array}$ & $\begin{array}{l}-0,83 \\
(0,48)\end{array}$ & $\begin{array}{l}-0,90 \\
(0,11)\end{array}$ & $\begin{array}{c}-0,84 \\
(0,01)\end{array}$ & $\begin{array}{l}-0,83 \\
(0,25)\end{array}$ \\
\hline Ala-pívot & $\begin{array}{l}-1,40 \\
(0,76)\end{array}$ & $\begin{array}{l}-0,59 \\
(0,09)\end{array}$ & $\begin{array}{l}-1,24 \\
(0,50)\end{array}$ & $\begin{array}{c}-1,23 \\
(0,22)\end{array}$ & $\begin{array}{c}-0,77 \\
(0,08)\end{array}$ & $\begin{array}{l}-0,92 \\
(0,13)\end{array}$ & $\begin{array}{l}-0,70 \\
(0,18)\end{array}$ & $\begin{array}{l}-0,87 \\
(0,66)\end{array}$ & $\begin{array}{l}-0,74 \\
(0,35)\end{array}$ & $\begin{array}{l}-0,95 \\
(0,64)\end{array}$ & $\begin{array}{c}-0,94 \\
(0,36)\end{array}$ \\
\hline Pívot & $\begin{array}{l}-0,76 \\
(0,33)\end{array}$ & $\begin{array}{l}-0,90 \\
(0,16)\end{array}$ & $\begin{array}{l}-0,90 \\
(0,03)\end{array}$ & $\begin{array}{c}-0,75 \\
(0,01)\end{array}$ & $\begin{array}{l}-0,68 \\
(0,08)\end{array}$ & $\begin{array}{c}-0,93 \\
(0,11)\end{array}$ & $\begin{array}{c}-0,43 \\
(---)\end{array}$ & $\begin{array}{l}-0,87 \\
(0,14)\end{array}$ & $\begin{array}{l}-0,58 \\
(0,21)\end{array}$ & $\begin{array}{c}-0,94 \\
(0,06)\end{array}$ & $\begin{array}{c}-0,77 \\
(0,11)\end{array}$ \\
\hline
\end{tabular}

Figura 1. Pérdidas de peso (Media \pm Desviación Estándar) en cada una de las participantes en el estudio a lo largo de los 10 partidos estudiados.

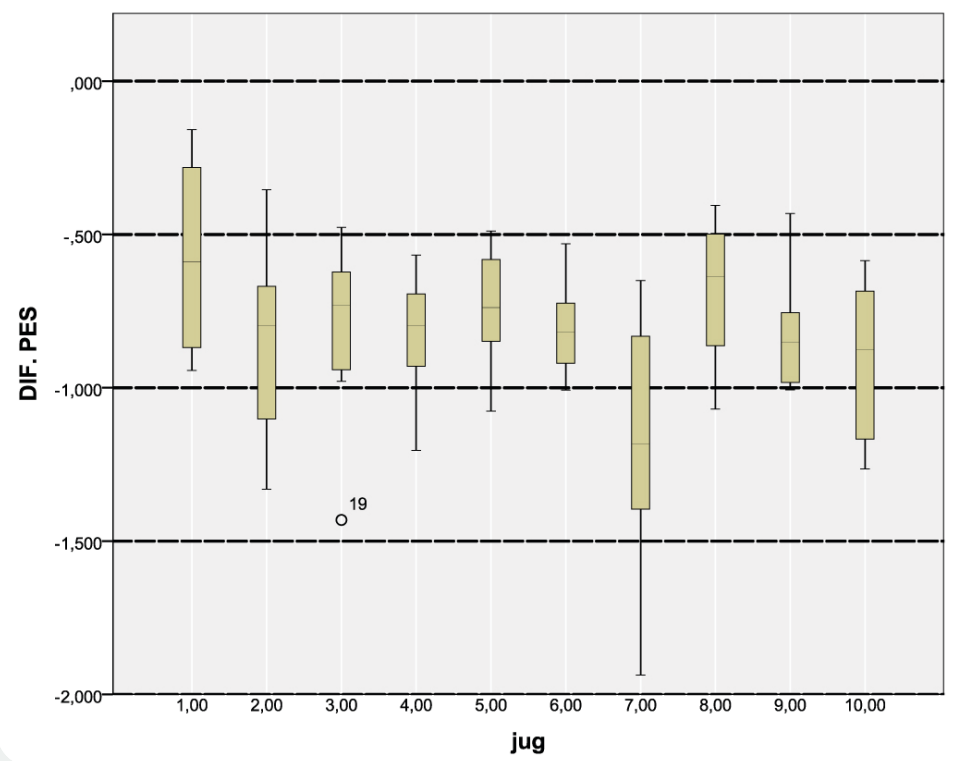

Ante esta problemática, diversos autores se han preocupado de analizar las necesidades hídricas en diversos deportes colectivos. Así por ejemplo, Barbero et al. ${ }^{14}$, utilizando un protocolo muy similar al utilizado en nuestro estudio, analizaron el balance hídrico en jugadores de fútbol sala en si- tuación real de partido, constatando que aquellos jugadores que jugaban más del $50 \%$ de la duración total del partido, perdían una media de $1,5 \pm 0,9 \%$ del $P C$, mientras que aqueIlos jugadores que jugaban menos del $50 \%$ del tiempo total perdían una media del $0,4 \pm 0,5 \%$ del $P C$. 
El baloncesto es un deporte colectivo de colaboración y oposición que se caracteriza por la existencia de fluctuaciones continuas en los niveles de intensidad, lo que hace que sea considerado como un deporte de intensidad elevada e intermitente ${ }^{15,16}$. Además, diversos estudios han constatado cómo la deshidratación puede disminuir la cantidad de lanzamientos realizados a canasta durante un partido y el nivel de precisión en los gestos técnicos específicos y en los lanzamientos de tiro libre ${ }^{17}$. En esta línea, Solera ${ }^{17}$ analizó el efecto del nivel de deshidratación en la eficacia del tiro libre, constatando cómo aquellos jugadores que no se rehidrataban al realizar un entrenamiento de baloncesto, alcanzaron pérdidas mayores $(2,90 \% \pm 0,87 \%$ del $P C)$, frente a los jugadores que utilizaban diferentes pautas de rehidratación $(1,26 \pm 0,88 \%$ del PC y $1,45 \pm 0,83 \%$ del PC)

Por su parte, Brandenburg y Gaetz ${ }^{18}$ analizaron las modificaciones de peso corporal durante dos partidos de baloncesto femenino, observando pérdidas del $0,7 \pm 0,8 \%$ del PC y $0,6 \pm 0,6 \%$ del $P C$, respectivamente. Por otro lado, en el mismo estudio se diferenció entre la pérdida de masa corporal durante el calentamiento de la sufrida durante el partido. Los valores de la ingesta de líquidos registrados durante el calentamiento se situaron en $0,35 \pm 0,2 \mathrm{~L}$ y en $0,25 \pm 0,1 \mathrm{~L}$, mientras que los registrados durante el partido ascendieron a $1,22 \pm 0,5 \mathrm{~L}$ y $1,40 \pm 0,6 \mathrm{~L}$, respectivamente. Todo ello sugiere que el simple calentamiento ya provoca un cierto grado de deshidratación y que por tanto resultaría interesante instaurar protocolos de rehidratación durante el calentamiento que permitiesen disminuir estos efectos, garantizando que el jugador no iniciara el partido en deuda hídrica. Resultados similares también fueron constatados por Osterberg et al. ${ }^{19}$ en jugadores masculinos, en los que se observaron pérdidas del 1,4\% del PC. Ziv y Lidor ${ }^{16}$ indicaron que la deshidratación en el baloncesto es debida a la repetición de esfuerzos de alta intensidad durante todo el partido y al elevado nivel de exigencia durante los entrenamientos. Además, los elevados niveles de humedad relativa alcanzados en algunos partidos contribuyen a aumentar la deshidratación, fruto de un incremento en el ratio de sudoración.

Todos estos datos son similares a los obtenidos en nuestra investigación, en la que se observaron niveles de pérdida de peso corporal que fluctuaron entre el $0,90 \%$ y $1,39 \%$ del PC, siendo mayores en aquellas jugadoras con más carga de minutos, lo que explica las diferencias observadas entre jugadoras al analizar las pérdidas hídricas sufridas (Figura 1). Pese a estas similitudes, en nuestro estudio se dieron ciertas circunstancias que deben ser tenidas en consideración. En primer lugar, los sujetos participantes en este estudio fueron mujeres. Se ha constatado un menor rango de sudoración en las mujeres debido a diversos factores como su menor tamaño corporal, un menor ratio de sudoración de sus glándulas sudoríparas debido a un umbral más elevado, a la existencia de diferencias hormonales en relación a los hombres (menores niveles de testosterona), a un inicio de la sudoración más tardío y a una menor evaporación en situaciones de humedad ${ }^{20-22}$. Además, el nivel de sudoración es distinto en función del momento del ciclo menstrual en el que éstas se encuentren ${ }^{20}$. Todo ello hace que se considere que las mujeres poseen mecanismos termorreguladores menos eficientes que los hombres ${ }^{23}$, lo que las condiciona también a tener una mayor temperatura corporal ${ }^{24}$. En segundo lugar, al tratarse de un partido de fase eliminatoria, es esperable que el factor emocional pudiese provocar un aumento en la frecuencia respiratoria, lo que podría incrementar la tasa de deshidratación fruto del vapor expulsado durante la respiración ${ }^{25,26}$. Por otro lado, no se observaron diferencias significativas en relación a la posición de juego, lo que sugiere que los niveles de deshidratación parecen ser independientes en relación a esta variable. Posiblemente, sean necesarios futuros estudios en los que se considere no sólo la posición sino también el nivel de intensidad y la distancia recorrida.

En relación al tipo de líquido ingerido, diversos autores sugieren la necesidad de utilizar bebidas que contengan agua, electrolitos y carbohidratos con la intención de compensar las pérdidas sufridas durante la práctica deportiva y mantener unos niveles de homeostasis óptimos, especialmente cuando la práctica deportiva ${ }^{27}$ se lleva a cabo en condiciones meteorológicas adversas. Por otro lado, el mantenimiento de los niveles de glucosa sanguínea durante la competición resulta fundamental en aquellos deportes en los que se requieren grandes niveles de coordinación, precisión y concentración; ya que nuestro cerebro consume como combustible principal glucosa (alrededor del $20 \%$ de la glucosa total consumida por nuestro organismo $)^{28}$. Desafortunadamente, la ingestión de bebidas con elevadas concentraciones de glucosa puede tener efectos negativos en la capacidad de hidratación, fruto de la disminución de los volúmenes plasmáticos como consecuencia de la necesidad de garantizar un nivel de fluido intracelular óptimo ${ }^{29}$. Todo ello sugiere la necesidad de ingerir bebidas de carácter isotónico que garanticen una correcta homeostasis. En nuestro estudio, sorprende el hecho de que la muestra analizada sólo consumía agua durante y después del partido, lo que indica un bajo nivel de conocimiento sobre las pautas básicas de hidratación y justifica la introducción de programas de formación que permitan mejorar las pautas de hidratación durante la práctica deportiva.

Por último, nos gustaría destacar que en este estudio no se valoró el nivel de hidratación previa a la competición con otros tipos de metodologías como el análisis de orina (osmolaridad, gravedad específica y color), o sangre, o la cuantificación de los sustratos presentes en el sudor o en la piel, debido al deseo y la necesidad de ser lo menos invasivos posible. Considerando la situación de estrés precompetitivo (no olvidemos que en esta competición las jugadoras se jugaban su permanencia en la categoría) el equipo investigador, de forma consensuada con los técnicos del equipo, 
tomó la decisión de valorar el peso corporal únicamente antes y después del partido con la intención de ser lo menos invasivos posible. Por otro lado, la necesidad de proponer herramientas sencillas y de bajo coste económico que puedan ser utilizadas por equipos de cualquier nivel, también contribuyó a la selección de la metodología utilizada.

\section{CONCLUSIONES}

En este estudio se constata la pérdida de peso durante el transcurso de la competición como reflejo de la deshidratación, siendo ésta una medida no invasiva que permite la elaboración de protocolos de hidratación que permitan optimizar el rendimiento y prevenir la aparición de lesiones. Dichos protocolos deberán ser siempre individualizados, ya que las necesidades hídricas variarán en función de cada deportista y del contexto en que se desarrolle la performance deportiva. Además, considerando que la mayoría de bebidas orientadas a la rehidratación se basan en bebidas glucosadas en las que se añaden electrolitos y sustancias potenciadoras del rendimiento, y teniendo en cuenta que la variación en la proporción de estas sustancias puede tener efectos distintos en el proceso de rehidratación, deberían considerarse, no sólo las cantidades de líquido ingerido, sino también la proporción de sustancias disueltas en él.

\section{$\longrightarrow$ CONFLICTO DE INTERESES}

Los autores expresan que no hay conflictos de interés al redactar el manuscrito.

\section{REFERENCIAS}

(1) Buono MJ, Sjoholm NT. Effect of physical training on peripheral sweat production. J Appl Physiol. 1988; 65(2): 811-4.

(2) Calvo B, García JM, Fernandes L. Análisis de la deshidratación en las diferentes etapas de entrenamiento en mujeres: Lucha vs. Judo. REYTE. 2014; 3(2): 31-41.

(3) Casa DJ, Armstrong LE, Hillman SK, Montain SJ, Reiff RV, Rich BS, et al. National athletic trainers' association position statement: fluid replacement for athletes. ] Athl Train. 2000; 35(2): 212-24.

(4) Cheuvront SN, Kenefick RW, Montain SJ, Sawka MN. Mechanisms of aerobic performance impairment with heat stress and dehydration. J Appl Physiol. 2010; 109(6): 1989-95.

(5) Macaluso F, Di Felice V, Boscaino G, Bonsignore G, Stampone $T$, Farina $F$, et al. Effects of three different water temperatures on dehydration in competitive swimmers. Science \& Sports. 2011; 26(5): 265-71.

(6) Flores-Salamanca R, Aragón-Vargas LF. Postexercise rehydration with beer impairs fluid retention, reaction time, and balance. Appl Physiol Nutr Metab. 2014; 39(10): 1175-81.

(7) American College of Sports Medicine, Sawka MN, Burke LM, Eichner ER, Maughan RJ, Montain S], et al. American College of Sports Medicine position stand. Exercise and fluid replacement. Med Sci Sports Exerc. 2007; 39(2): 377-90.

(8) Robertson GL. Vaptans for the treatment of hyponatremia. Nat Rev Endocrinol. 2011; 7(3): 151-61.

(9) Cox GR, Broad EM, Riley MD, Burke LM. Body mass changes and voluntary fluid intakes of elite level water polo players and swimmers. J Sci Med Sport. 2002; 5(3): 183-93.

(10) Melin B, Curé M, Pequignot JM, Bittel J. Body temperature and plasma prolactin and norepinephrine relationships during exercise in a warm environment: effect of dehydration. Eur ] Appl Physiol Occup Physiol. 1988; 58(1-2): 146-51.

(11) Holway FE, Spriet LL. Sport-specific nutrition: practical strategies for team sports. J Sports Sci. 2011; 29 Suppl 1: S115-125.

(12) Kovacs MS. A review of fluid and hydration in competitive tennis. Int J Sports Physiol Perform. 2008; 3(4): 413-23.

(13) Cheuvront SN, Montain SJ, Sawka MN. Fluid replacement and performance during the marathon. Sports Med. 2007; 37(4-5): 353-7.

(14) Barbero JC, Castagna C, Granda J. Deshidratación y reposición hídrica en jugadores de fútbol sala: efectos de un programa de intervención sobre la pérdida de líquidos durante la competición. Mot Eur J Hum Mov. 2006; 17: 93-106.

(15) Castagna C, Impellizzeri FM, Chaouachi A, Ben Abdelkrim N, Manzi V. Physiological responses to ball-drills in regional level male basketball players. J Sports Sci. 2011; 29(12): 1329-36.

(16) Ziv G, Lidor R. Physical attributes, physiological characteristics, on-court performances and nutritional strategies of female and male basketball players. Sports Med. 2009; 39(7): 547-68.

(17) Solera A. Efectos de la deshidratación y la rehidratación en la efectividad del tiro libre de baloncesto. Pensar en Movimiento: Revista de Ciencias del Ejercicio y la Salud. 2003; 3(1): 35-42.

(18) Brandenburg JP, Gaetz M. Fluid Balance of Elite Female Basketball Players Before and During Game Play. IJSNEM. 2012; 22(5): 347-52.

(19) Osterberg KL, Horswill CA, Baker LB. Pregame urine specific gravity and fluid intake by National Basketball Association players during competition. J Athl Train. 2009; 44(1): 53-7.

(20) Inoue Y, Tanaka Y, Omori K, Kuwahara T, Ogura Y, Ueda H. Sex -and menstrual cycle-related differences in sweating and cutaneous blood flow in response to passive heat exposure. Eur ] Appl Physiol. 2005; 94(3): 323-32.

(21) Ichinose-Kuwahara T, Inoue Y, Iseki Y, Hara S, Ogura Y, Kondo $N$. Sex differences in the effects of physical training on sweat gland responses during a graded exercise. Exp Physiol. 2010; 95(10): 1026-32.

(22) Gagnon D, Kenny GP. Sex modulates whole-body sudomotor 
thermosensitivity during exercise. J Physiol. 2011; 589(Pt 24): 6205-17.

(23) Shapiro Y, Pandolf KB, Avellini BA, Pimental NA, Goldman RF. Physiological responses of men and women to humid and dry heat. J Appl Physiol Respir Environ Exerc Physiol. 1980; 49(1): 1-8.

(24) Bittel J, Henane R. Comparison of thermal exchanges in men and women under neutral and hot conditions. J Physiol. 1975; 250(3): 475-89.

(25) Henry M, de las Cuevas C, González JL, Gracia R. Relevancia de factores psicosociales en el aparato respiratorio. Psiquis. 1991; 12: 244-7.

(26) Delistraty DA, Greene WA, Carlberg KA, Raver KK. Use of graded exercise to evaluate physiological hyperreactivity to mental stress. Med Sci Sports Exerc. 1991; 23(4): 476-81.

(27) Burke LM. Nutritional needs for exercise in the heat. Comp Biochem Physiol, Part A Mol Integr Physiol. 2001; 128(4): 73548.

(28) Godoy DA, Behrouz R, Di Napoli M. Glucose control in acute brain injury: does it matter? Curr Opin Crit Care. 2016; 22(2): 120-7.

(29) Allen S, Miller KC, Albrecht J, Garden-Robinson J, BlodgettSalafia E. Ad libitum fluid intake and plasma responses after pickle juice, hypertonic saline, or deionized water ingestion. J Athl Train. 2013; 48(6): 734-40. 